\title{
Liposomal honokiol inhibits glioblastoma growth through regulating macrophage polarization
}

\author{
Shenglan $\mathrm{Li}^{1}$, Long $\mathrm{Li}^{2}$, Jinyi Chen ${ }^{1}$, Yaqiong Fan ${ }^{1}$, Ce Wang ${ }^{1}$, Yuan $\mathrm{Du}^{3}$, Caixia Guo ${ }^{2}$, Feng Chen ${ }^{1}$, \\ Wenbin $\mathrm{Li}^{1}$ \\ ${ }^{1}$ Department of Neuro-Oncology, Cancer Center, Beijing Tiantan Hospital, Capital Medical University, Beijing, China; ${ }^{2}$ Department of Cardiology, \\ Beijing Tiantan Hospital, Capital Medical University, Beijing, China; ${ }^{3}$ School of Basic Medicine Science, Jiamusi University, Jiamusi, China \\ Contributions: (I) Conception and design: F Chen, C Guo, W Li; (II) Administrative support: W Li; (III) Provision of study materials or patients: \\ Y Du, C Wang; (IV) Collection and assembly of data: J Chen, Y Fan; (V) Data analysis and interpretation: S Li, L Li; (VI) Manuscript writing: All \\ authors; (VII) Final approval of manuscript: All authors. \\ Correspondence to: Wenbin Li, MD; Feng Chen, MD. Department of Neuro-Oncology, Cancer Center, Beijing Tiantan Hospital, Capital Medical \\ University, No. 119, Nansihuan West Road, Fengtai District, Beijing 100070, China. Email: liwenbin@ccmu.edu.cn; chenfeng@bjtth.org; \\ Caixia Guo, MD. Department of Cardiology, Beijing Tiantan Hospital, No. 119, South 4th Ring West Road, Fengtai District, Beijing 100070, \\ China. Email: cxgbb@163.com.
}

Background: Glioblastoma is a type of aggressive brain tumor-related to infiltrating microglia/ macrophages. Various studies have identified antitumor properties of a bioactive plant compound named honokiol, originating from the Magnolia species. This beneficial characteristic of honokiol has been discovered in many malignant tumors.

Methods: We investigated the molecular mechanisms behind the anti-glioma effects of liposomal honokiol (Lip-HNK) using qRT-PCR, Western blot, co-culture, and in vivo animal experiments.

Results: It was discovered that the expression of M1 markers such as CD11c, inducible nitric oxide synthase (iNOS), and major histocompatibility complex (MHC) II (IA/IE subregions) induced by lipopolysaccharide (LPS)/IFN- $\gamma$ was increased by Lip-HNK, and M2 markers Arg1 and CD206 induced by interleukin (IL)-4 had reduced expression, thus inhibiting tumor cell growth through co-culture experiments. After Lip-HNK treatment, a considerable increase in signal transducer and activator of transcription 1 (STAT1) activation was observed, and in contrast, STAT6 activation was suppressed. STAT1 and STAT6 are the key signaling molecules mediating M1 and M2 polarization, respectively. Furthermore, the percentage of CD11c-positive M1 macrophages was increased by Lip-HNK in G422 xenograft mice, while Lip-HNK treatment reduced the CD206-positive M2 macrophage distribution in tumor tissues. These findings are consistent with the decline in tumor volume seen in mice treated with Lip-HNK.

Conclusions: Lip-HNK inhibits the growth of glioblastoma by upregulating M1 macrophages and limiting M2 phenotypic macrophages.

Keywords: Glioblastoma (GBM); liposomal honokiol; macrophage; polarization

Submitted Apr 13, 2021. Accepted for publication Aug 02, 2021.

doi: $10.21037 / \mathrm{atm}-21-1836$

View this article at: https://dx.doi.org/10.21037/atm-21-1836

\section{Introduction}

Gliomas account for $29 \%$ of all brain tumors, among which glioblastoma [GBM, World Health Organization (WHO) grade IV] is the most malignant, and the average survival time post-diagnosis is only 15 months (1). The treatment of GBM depends on maximum surgical resection, followed by radiotherapy and temozolomide combined with chemotherapy. However, recurrence is inevitable, and the 


\section{Page 2 of 13}

prognosis is still poor. Therefore, there is an urgent call for new treatment options, including immunotherapy.

The microenvironment of GBM includes innate immune cells, which are recognized as glioma-associated microglia/ macrophages (GAMs) (2). There are 2 different activation states of GAMs: $M 1$ is the tumor inhibition state, M2 is the tumor supportive state, promoting tumor growth. It is believed that the difference between phenotypes reflects a series of plastic functional conditions rather than discrete activation states $(3,4)$.

M1 macrophages are characterized by the expression of inducible nitric oxide synthase (iNOS) and have the ability to phagocytose and activate pro-inflammatory synthesis, thereby playing an antitumor role. Macrophages can polarize into the M1 phenotype under the action of a variety of pro-inflammatory cytokines, such as interferon- $\gamma$ (IFN- $\gamma$ ) and lipopolysaccharide (LPS) (5). On the contrary, M2 is characterized by arginase-1 (Arg1) activity, which promotes tumor development through tissue remodeling, cell proliferation, angiogenesis, and immune regulation (6). Results showed that the reversal of M2 to M1 had a significant beneficial effect on the growth of gliomas (7). However, while the interaction between GAMs and GBM cells has been established, the role of this interaction in the progression of GBM remains unclear.

Honokiol (HNK), extracted from the bark and cones of Magnolia officinalis, is small bisphenol lignin. In recent years, HNK has attracted extensive attention due to its superior antitumor effects in various tumor cells and animal models. As an antitumor drug, the molecular mechanisms of HNK have been elucidated, including the induction of apoptosis and cell cycle arrest, and the inhibition of tumor invasion and metastasis through suppressing the epidermal growth factor receptor (EGFR), nuclear factor- $\mathrm{\kappa B}$ (NF$\kappa \mathrm{B})$, RAS/extracellular signal-regulated kinase (ERK), and phosphoinositide 3-kinase (PI3K)/protein kinase B (AKT)/ mammalian target of rapamycin (mTOR) pathways (8-10). However, the clinical application of HNK has been largely limited due to its low bioavailability and poor water solubility. Therefore, overcoming these disadvantages of HNK has become imperative, and one solution is to package it in suitable nanocarriers. Liposomes have the most promising clinical application. In general, liposomes are used to increase the solubility of drugs by carrying them in lipid bilayers (11). Therefore, the subsequent in vitro and in vivo inhibitory effects of liposomal honokiol (Lip-HNK) on tumor cell growth can be investigated.
The primary objective of this study was to investigate the mechanism of Lip-HNK in mediating macrophage polarization in the progression of GBM. In addition, the antitumor effect of Lip-HNK was confirmed in vivo.

We present the following article in accordance with the ARRIVE reporting checklist (available at https://dx.doi. org/10.21037/atm-21-1836).

\section{Methods}

Experiments were performed under a project license (No. KYSQ 2021-021-01) granted by the Committee on the Ethics of Animal Experiments of Beijing Tiantan Hospital, in compliance with Chinese national and institutional guidelines for the care and use of animals.

\section{Chemicals and reagents}

Chengdu Jinrui Biotechnology Company (Chengdu, China) provided Lip-HNK ( $>98 \%$ purity). LPS, IFN- $\gamma$, and interleukin (IL)-4 were purchased from Sigma-Aldrich (St. Louis, MO, USA).

\section{Cell culture and drug treatment}

The U87 primary human GBM cell line expressing luciferase and red fluorescent protein (U87-RFP-Luc) was purchased from Keyuandi, Biological Technology Development Co., Ltd. (Shanghai, China). The LN229 cell line marked with a green fluorescent protein (LN229GFP) was purchased from the Institute of Medical Sciences, Peking Union Medical College (Beijing, China). The macrophage cell line RAW264.7 and the microglia cell line BV2 derived from mice were obtained from the American Type Culture Collection (ATCC). Cell lines were cultured in DMEM medium with $10 \%$ (vol/vol) fetal bovine serum (FBS), $100 \mu \mathrm{g} / \mathrm{mL}$ streptomycin, and 100 units $/ \mathrm{mL}$ penicillin and were placed in an incubator containing 5\% carbon dioxide at a temperature of $37^{\circ} \mathrm{C}$. Lip-HNK was dissolved in sterile water, and the cells were directly exposed to it and/or other reagents indicated herein at different intervals and at different concentrations. Polarization of M1 macrophages was achieved by stimulating cells with $20 \mathrm{ng} / \mathrm{mL}$ IFN- $\gamma$ and $10 \mathrm{ng} / \mathrm{mL}$ LPS. Polarization of M2 macrophages was achieved by stimulating cells with $20 \mathrm{ng} / \mathrm{mL}$ IL-4. Lip-HNK was added after M1 inducer LPS/IFN- $\gamma$ or M2 inducer IL-4 treatment for 24 hours. 


\section{Determination of total nitric oxide (NO)}

The culture solution of RAW264.7 cells was collected to measure NO concentration using the nitrate reductase method with a kit. Nitrate reductase can turn NO-derived nitrate into nitrite. The absorbance of nitrite was analyzed by a spectrophotometer set at $550 \mathrm{~nm}$. The concentration of $\mathrm{NO}$ was calculated as follows: concentration of $\mathrm{NO}(\mu \mathrm{M})$ $=($ absorbance of treated wells - absorbance of blank wells $) /$ (absorbance of standard wells - absorbance of blank wells) $\times$ standard concentration $(20 \mu \mathrm{M})$.

\section{Measurement of tumor necrosis factor (TNF)- $\alpha$}

The concentrations of TNF- $\alpha$ in supernatants were quantified with enzyme linked immunosorbent assay (ELISA) by utilizing commercially available antibodies, according to the protocol provided by the supplier.

\section{Extraction of $R N A$ and quantitative real-time PCR (qRT-PCR)}

Total RNA extraction was conducted using the RNA-Quick Purification Kit from macrophage cell lines by Yishan Biotechnology Co., Ltd. (Shanghai, China). Using the Transcriptor cDNA Synth.kit2 by Roche Biotechnology Co., Ltd. (Shanghai, China), the RNA was then reversetranscribed to cDNA. With the use of SYBR Green PCR Master Mix (Washington, UK), real-time PCR with the sequence detection system ABI PRISM 7900 (PerkinElmer, Branchburg, NJ, USA) was conducted. mRNA expression was normalized to glyceraldehyde-3-phosphate dehydrogenase (GAPDH), and the results were displayed as a relative figure established by the calculation of $2^{-\Delta \Delta C t}$. The results were obtained by means of the comparative $\mathrm{Cq}$ method $\left(2^{-\Delta \Delta C q}\right)$ using GAPDH as an internal control. The primer sequences used in the qRT-PCR analysis are shown in Table S1.

\section{Expression of macrophage markers detected by flow cytometry}

BV2 and RAW264.7 cell lines were blocked with $3 \%$ bovine serum albumin (BSA) for $45 \mathrm{~min}$, then stained with a CD11c antibody, major histocompatibility complex (MHC) class II subunits IA-IE antibody, CD206 antibody, or F4/80 antibody (Cat. \#117311, \#107626, \#141708, and \#123118, respectively, BioLegend, San Diego, CA, USA).
The processed samples were assessed with CytoFLEX S (Beckman Coulter, USA). The Flow Jo software package was used to analyze the proportion of positive cells.

\section{Co-culture of tumor cells with macrophages}

LN229-GFP and U87-RFP-Luc human GBM cells were cultured individually or co-cultured directly with BV2 or RAW264.7 cells as densities of $1: 10$ or $1: 5$ in a 6 -well plate. After 12 hours, the cells were treated either with 2.5, 5 , or $10 \mu \mathrm{M}$ of Lip-HNK alone, $\mathrm{M} 1$ inducer $(10 \mathrm{ng} / \mathrm{mL}$, LPS; $20 \mathrm{ng} / \mathrm{mL}$, IFN- $\gamma$ ), or M2 inducer (20 ng/mL, IL-4) with or without Lip-HNK for a further 48 hours. Using the fluorescence microscope, the features of tumor cells were observed, then cells were incubated with $15 \mu \mathrm{g} / \mathrm{mL}$ luciferase for $5 \mathrm{~min}$. With the aid of the EnSpire Multimode Plate Reader (PerkinElmer, Inc, USA), the bioluminescence of GBM cells was also evaluated.

\section{Western blot analysis}

Cells were lysed with RIPA buffer containing a mixture of phosphatase and protease inhibitors (Thermo Fisher Scientific, Waltham, Massachusetts, USA). The BCA ${ }^{\mathrm{TM}}$ Protein Assay Kit (Fremont Thermal Science, California, USA) was utilized to quantify protein concentration. The proteins were isolated using sodium dodecyl sulfatepolyacrylamide gel electrophoresis (SDS-PAGE), and bands were transferred onto a polyvinylidene fluoride (PVDF) membrane. The membrane was incubated overnight with the primary antibody, blocked with $5 \%$ milk at $37{ }^{\circ} \mathrm{C}$ for $60 \mathrm{~min}$, then incubated with the secondary antibody. Finally, the ECL system (Perkin Elmer, Waltham, MA, USA) was used to detect immune complexes. Antibodies against the following proteins were used: phosphor-STAT1 $1^{\text {Tyr701, }}$ phosphor-STAT1 $1^{\text {ser727 }}$, STAT1, STAT6, phosphorSTAT6 ${ }^{\text {Tyr641 }}$, and GAPDH (Cat. \#8826, \#9167, \#14994, \#56554, \#5397, and \#5174, respectively, Cell Signaling Technology, USA). The secondary antibodies were from Abcam (Cambridge, UK).

\section{G422 GBM cell xenograft in a murine model}

Sixteen female ICR mice aged 6 weeks, weighing 18-22 g, were acquired from Beijing Wei Tong Li Hua Experimental Animal Technology Co., Ltd. Subcutaneous injection of G422 GBM cells $\left(1 \times 10^{6}\right)$ with $0.2 \mathrm{~mL}$ phosphate-buffered saline (PBS) was made in the right flank of the mice. On 
the second day following the implantation of the tumor cells, if these animals succeeded in tumorigenesis, they were included in the study, and mice were randomly allocated into 4 groups ( $\mathrm{n}=4$ in each group). Mice were intraperitoneally injected with 12.5 or $25 \mathrm{mg} / \mathrm{kg}$ LipHNK or liposomes (control group) every day for 3 weeks. Intraperitoneal injection of temozolomide $(50 \mathrm{mg} / \mathrm{kg})$ was administered to the positive control group for 5 days. The administration time was 8:30 a.m. every day. The administration order of each group was random, and the administration order of each animal in each group was random. Both the research team and the veterinary staff monitored animals twice daily. Health was monitored by weight (every 3 days), food and water intake, and general assessment of animal activity, panting, and fur condition. The maximum size the tumors were allowed to grow to in the mice before euthanasia was $2,000 \mathrm{~mm}^{3}$. At the end of the experiment, the mice were sacrificed, and the tumor was isolated, measured, and examined.

\section{Immunofluorescence}

The tumor tissues of mice injected with G422 tumor cells were frozen immediately in an Oct embedding medium and sectioned at a thickness of $8 \mu \mathrm{m}$. Tissues were incubated with mouse CD11c monoclonal antibody and rat anti-F4/80 to distinguish M1 macrophage markers (Cat. \#ab254183 and \#ab6640, respectively, Abcam, Cambridge, MA, USA). Frozen tissue sections were immunostained with rabbit anti-CD206 and rat anti-F4/80 antibodies (Cat. \#ab6640 and \#ab64693, respectively, Abcam, Cambridge, MA, USA) in order to analyze M2 phenotypic macrophages. Subsequently, the slides were stained with corresponding anti-mouse Alexa Fluor 488 dye (green), anti-rabbit Alexa Fluor 488 dye (green), or anti-rat Alexa Fluor 647 dye (red) (Invitrogen-Molecular Probes, Carlsbad, CA, USA) fluorescein-labeled secondary antibodies. Sections were treated with a blue nuclear counterstain 4',6-diamidino2-phenylindole (DAPI) for $5 \mathrm{~min}$ and visualized using an Olympus FV1000 confocal microscope. Quantitative analysis was carried out using Image-Pro Plus version 6.0 (media cybernetics, Rockville, Maryland, USA) to analyze positive cell numbers in 6 randomly selected fields from each section.

\section{Statistical analysis}

Using SPSS version 19.0 software (SPSS Inc., Illinois,
USA), one-way ANOVA was performed to analyze the experimental data, and the results were displayed as mean \pm standard deviation (SD). The statistical significance level was set at a $\mathrm{P}$ value $<0.05$.

\section{Results}

\section{Lip-HNK promoted M1 macrophage polarization induced by IFN $-\gamma$ and LPS}

The influence of Lip-HNK on the polarization of macrophages at concentrations ranging from 2.5 to $10 \mu \mathrm{M}$ was observed, and the results indicated that Lip-HNK did not affect macrophage proliferation at these doses (Figure S1A). Then, when exposed to Lip-HNK and IFN- $\gamma /$ LPS, iNOS mRNA was upregulated in a concentrationdependent manner in RAW264.7 cells, and the Arg1 mRNA level was significantly downregulated (Figure 1A). Similarly, in BV2 cells, compared with cells treated with IFN- $\gamma / \mathrm{LPS}$, the level of iNOS mRNA was substantially elevated by the combination of IFN- $\gamma / \mathrm{LPS}$ with Lip-HNK. In contrast, the level of Arg1 mRNA was lowered (Figure 1B). In order to investigate the specific responsibility of Lip-HNK in the differentiation of M1 macrophages, flow cytometry was used to detect cell surface markers. As illustrated in Figure $1 C, 1 D$, a dose-dependent increase in CD11c expression (Figure 1C) and MHC class II subunit IA/IE expression (Figure 1D) in RAW264.7 cells was observed after treatment with IFN- $\gamma / \mathrm{LPS}$ combined with Lip-HNK. These results suggest that Lip-HNK can promote M1 macrophage differentiation. In addition, we also detected TNF- $\alpha$ expression, and the results were consistent with the above findings (Figure S1).

\section{Lip-HNK inbibits IL-4-induced macrophage polarization to $M 2$}

We investigated the effect of Lip-HNK on macrophage M2 polarization induced by IL-4 in RAW264.7 cells. Lip-HNK decreased the expression of iNOS mRNA and increased Arg 1 mRNA expression induced by IL-4 in a dosedependent manner (Figure $2 A$ ). In BV2 cells treated with IL-4, similar iNOS and Arg1 mRNA levels were observed after Lip-HNK treatment (Figure 2B). In addition, IL4-induced CD206 expression was reduced in RAW264.7 (Figure 2C) and BV2 cells (Figure 2D) after treatment with Lip-HNK.

Macrophages, which are key cells in regulating 
A

RAW264.7

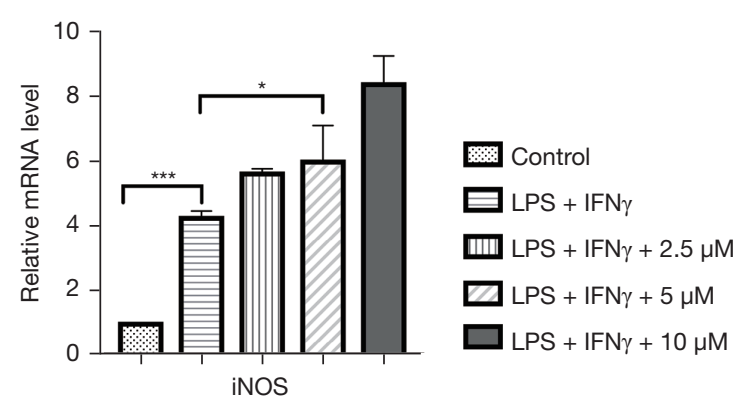

B

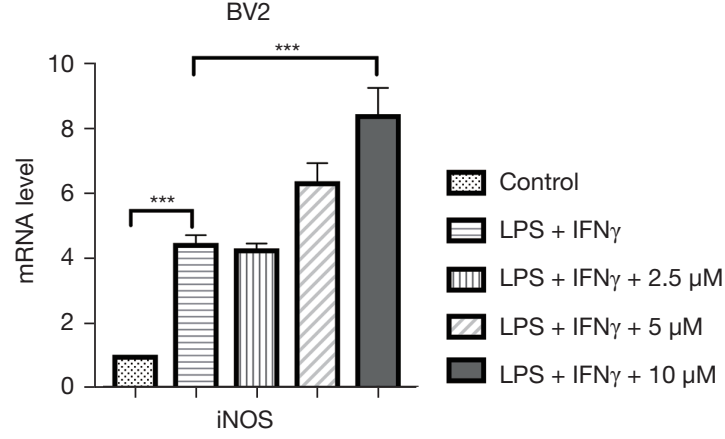

C

RAW264.7
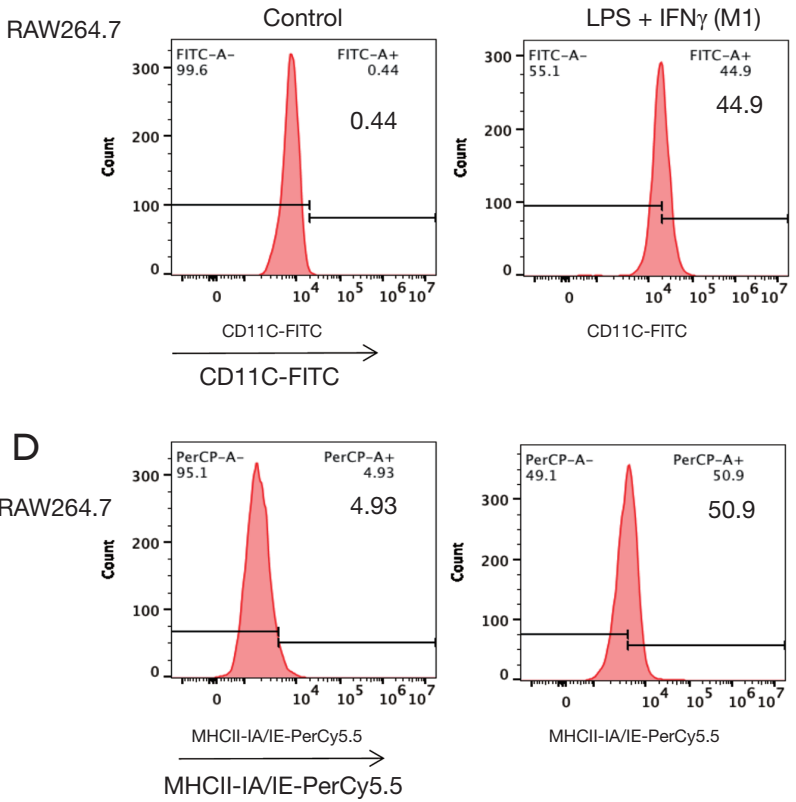

RAW264.7

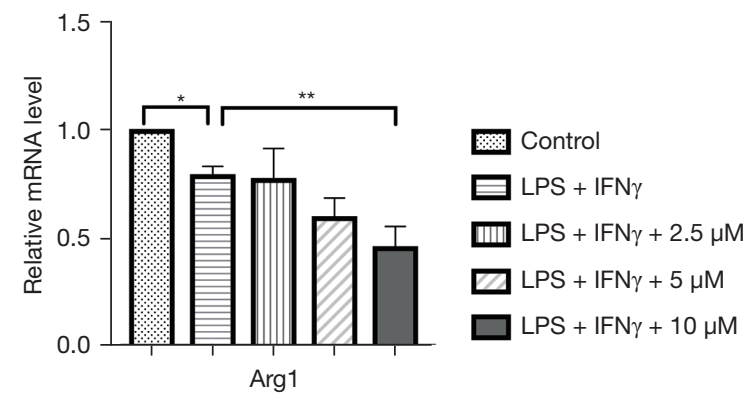

BV2

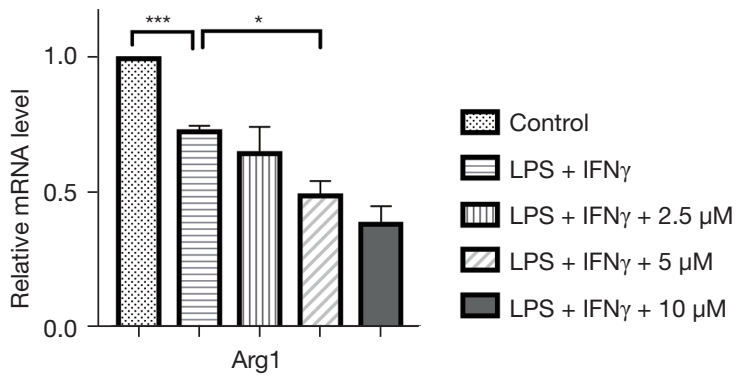

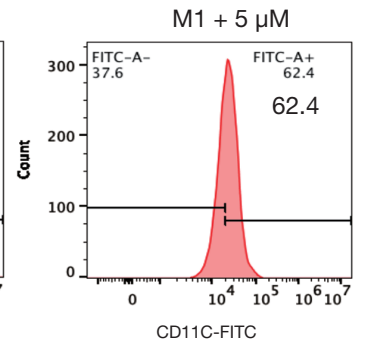
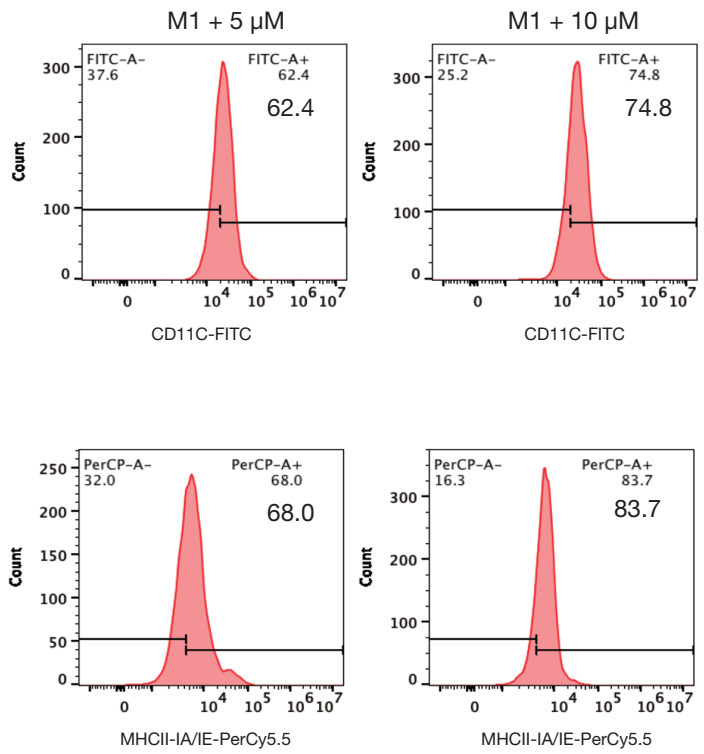

Figure 1 Effect of liposomal honokiol (Lip-HNK) on macrophage marker expression induced by lipopolysaccharide (LPS) and interferon $\gamma$ (IFN- $\gamma)$. Macrophages were treated with LPS $(10 \mathrm{ng} / \mathrm{mL})$ and IFN- $\gamma(20 \mathrm{ng} / \mathrm{mL})$ for 24 hours and Lip-HNK for 48 hours. M2-marker gene Arg1 and M1-marker gene inducible nitric oxide synthase (iNOS) mRNA levels in RAW264.7 (A) and BV2 cells (B) were detected by realtime RT-PCR, and then normalized to glyceraldehyde-3-phosphate dehydrogenase (GAPDH). (C,D) Flow cytometry was used to examine CD11c (C) and major histocompatibility complex (MHC) class II IA-IE (D) expression in RAW264.7 cells. The data are shown as mean \pm standard deviation. *, $\mathrm{P}<0.05 ;{ }^{* *}, \mathrm{P}<0.01 ;{ }^{* * *}, \mathrm{P}<0.001$. 

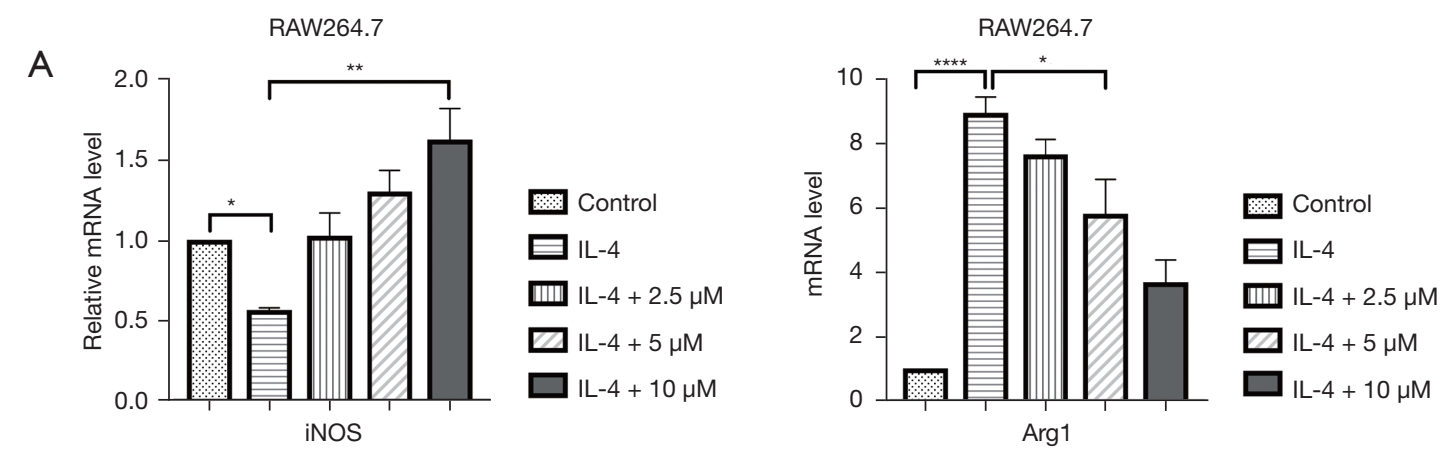

B
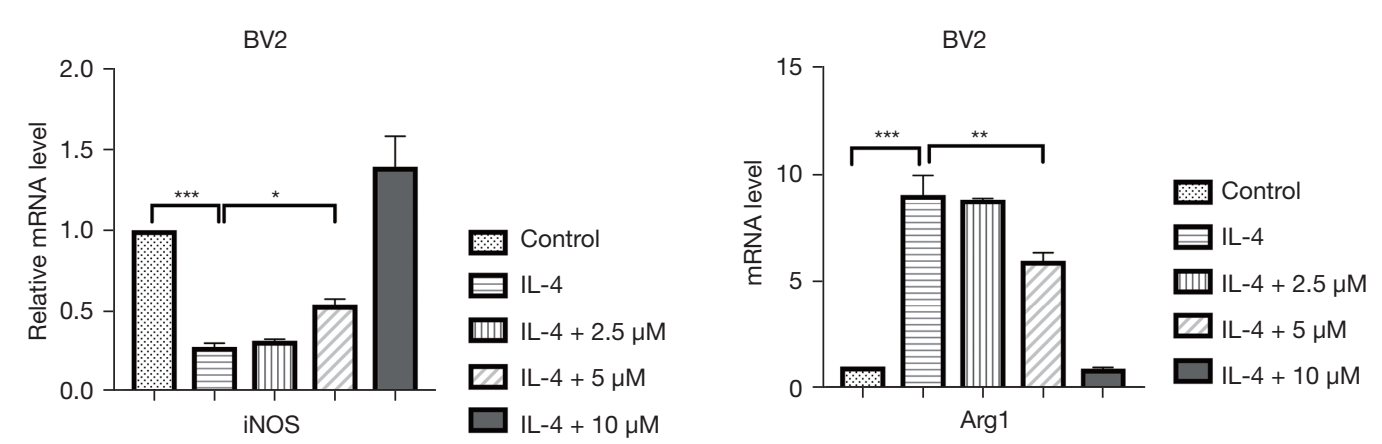

C

Control

IL-4 (M2)
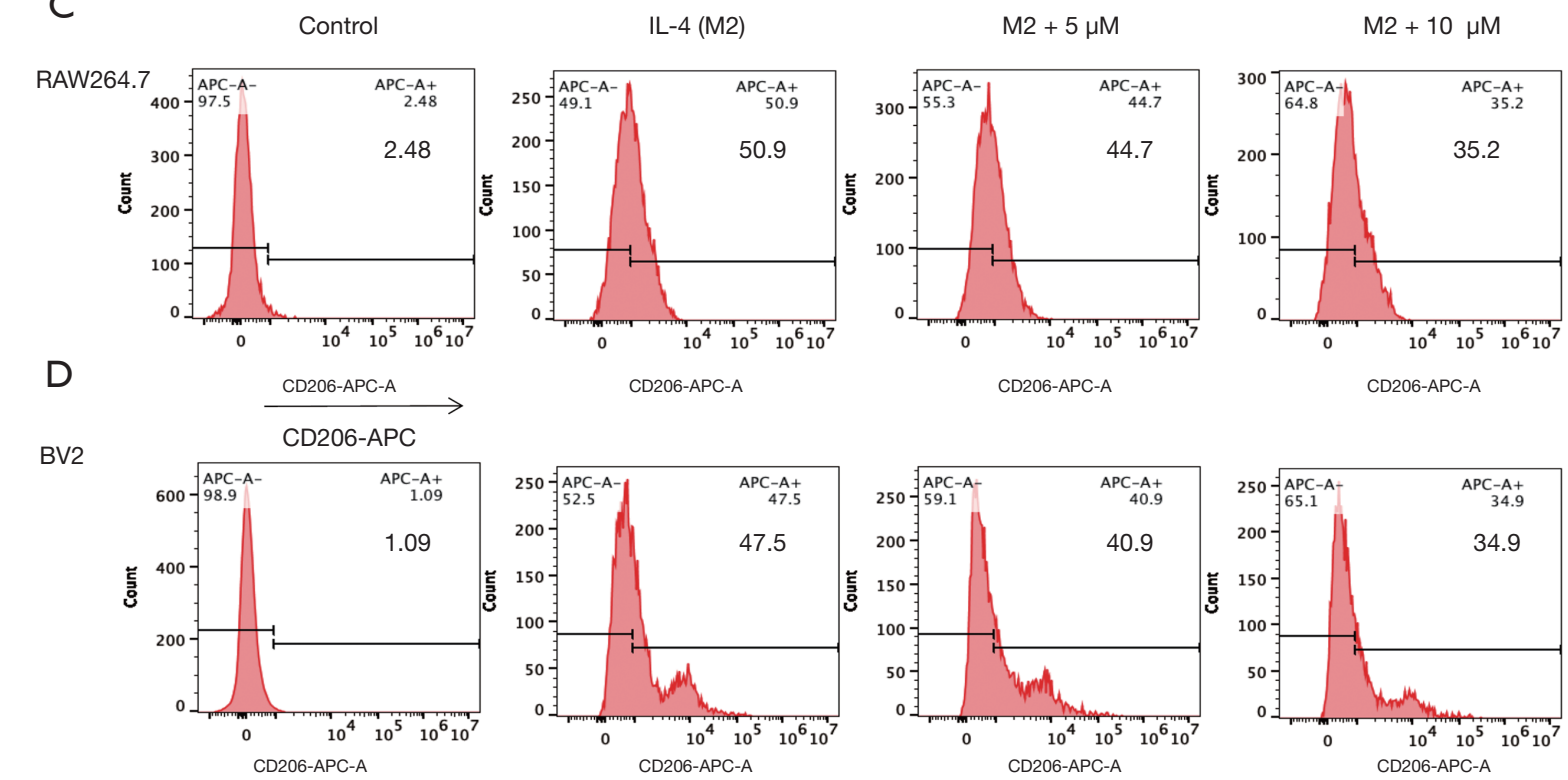

Figure 2 The effect of liposomal honokiol (Lip-HNK) on interleukin 4 (IL-4)-mediated macrophage marker expression. RAW264.7 and BV2 cells were treated with IL-4 and Lip-HNK of various concentrations for 48 hours. The mRNA levels of the M1 marker gene inducible nitric oxide synthase (iNOS) and the M2 marker gene Arg1 were detected by qRT-PCR in RAW264.7 cells (A) and BV2 cells (B). The expression of mRNA was standardized by glyceraldehyde-3-phosphate dehydrogenase (GAPDH). CD206 expressed in RAW264.7 (C), and BV2 cells (D) was assessed with flow cytometry. The 3 independent experiments are illustrated by histogram bars. The data are displayed as the mean \pm standard deviation. ${ }^{*}, \mathrm{P}<0.05 ;{ }^{* *}, \mathrm{P}<0.01 ;{ }^{* * *}, \mathrm{P}<0.001 ;{ }^{* * *}, \mathrm{P}<0.0001$. 
inflammatory responses, produce a wide range of inflammatory mediators, such as TNF- $\alpha$ or NO (12). Macrophages were stimulated to M2, and the release of M1 markers (NO and TNF- $\alpha$ ) in the culture medium was increased after Lip-HNK treatment, which further proves that Lip-HNK could repolarize cells (Figure S2A,S2B). In addition, we also found that the levels of IL-10 and transforming growth factor $\beta$ (TGF- $\beta$ ) in M2 polarized macrophages decreased after Lip-HNK treatment (Figure S2C,S2D). The results indicate that Lip-HNK can effectively inhibit the M2 phenotype differentiation of macrophages.

\section{Lip-HNK regulated M1/M2 macrophage polarization via the STAT1/6 pathway}

In order to comprehend the underlying molecular mechanism of Lip-HNK on macrophage polarization, the effects of Lip-HNK on the STAT pathway were investigated. First, the effects of Lip-HNK on M1 macrophage repolarization were studied. The activation of the STAT1 pathway by IFN- $\gamma$ is indispensable for the polarization of M1. We detected the protein level of STAT1/6 after treatment with Lip-HNK in macrophages stimulated by M1/M2 inducers. The results indicated that M1-inducer (LPS/IFN- $\gamma$ ) treatment strongly triggered STAT1, $\mathrm{p}($ Ser727)-STAT1, and $\mathrm{p}($ Tyr701)-STAT1 when RAW264.7 cells (Figure 3A) and BV2 (Figure 3B) cells were treated with Lip-HNK. Meanwhile, p-STAT6 (Tyr641) and total STAT6 were significantly reduced by combined IL-4 and Lip-HNK treatment in a dose-dependent manner in RAW264.7 (Figure 3C) and BV2 cells (Figure 3D). The results demonstrated a possible regulatory effect of LipHNK on M1/M2 polarization mostly through the STAT1 and STAT6 signaling pathways.

\section{Inbibition of tumor growth by Lip-HNK through interfering with the interactions between tumor cells and macrophages}

It was shown that Lip-HNK at its highest concentration in macrophage polarization did not influence tumor cell lines and macrophage proliferation directly (Figure S3A,S3B). An investigation on whether Lip-HNK regulates the interaction between brain tumor cells and macrophages was also performed. The proliferation of U87 cells was nearly unchanged by the regimen of prescribed concentrations of Lip-HNK, IFN- $\gamma / \mathrm{LPS}$, or IL-4 alone (Figure S4). In the co-culture environment, macrophage cell lines treated with the M2 inducer could induce the proliferation of U87 cells, and a substantial decrease in the growth of U87 cells cocultured with macrophages treated with the $M 1$ inducer was observed $(\mathrm{P} \leq 0.05)$ (Figure $4 A$ ). In addition, Lip-HNK treatment significantly inhibited the tumor-promotive function of M2 macrophages (Figure 4B). When LN229GFP cells were co-cultured with macrophages stimulated by M1/M2 inducers, a similar phenomenon was observed. Lip-HNK treatment also reduced the stimulating effect of M2 macrophages on the growth of tumors (Figure 4C,4D). These findings demonstrate that Lip-HNK plays an antitumor role by interfering with cell-to-cell interactions between tumor cells and macrophages.

\section{Lip-HNK inbibited the growth of GBMs in the murine model}

In order to explore the anticancer effect of Lip-HNK on GBMs, a G422 GBM xenograft murine model was established in vivo. As shown in Figure 5A,5B, LipHNK could inhibit tumor proliferation in G422bearing mice without losing body weight (Figure S5). In addition, the findings were supported by the results of immunofluorescence staining. In the tumor sections of mice treated with Lip-HNK, the number of M1 macrophages positive for CD11c and F4/80 increased (Figure 5C,5D). After treatment with Lip-HNK, a substantial decrease was seen in the number of $\mathrm{M} 2$ macrophages penetrating into tumor sections immunostained with F4/80 and CD206 (Figure 5E, $5 F$ ). Overall, these results suggest that Lip-HNK significantly reduces $\mathrm{M} 2$ macrophage infiltration into tumor tissues of G422 tumor-bearing mice, but increases the accumulation of M1 macrophages.

\section{Discussion}

Previous studies and the present study have shown that HNK exerts anticancer effects by inducing cell cycle arrest and apoptosis of tumor cells, as well as by impeding tumor invasion and metastasis (13-15). Because of its poor solubility, HNK is not widely used in clinical cancer treatment. However, liposomes can overcome this limitation; thus, Lip-HNK has promising clinical applications. The blood-brain barrier (BBB) restricts the entry of toxic substances, drug molecules, and various proteins and peptides to maintain brain homeostasis. One of the most important factors in drug delivery is selecting an 

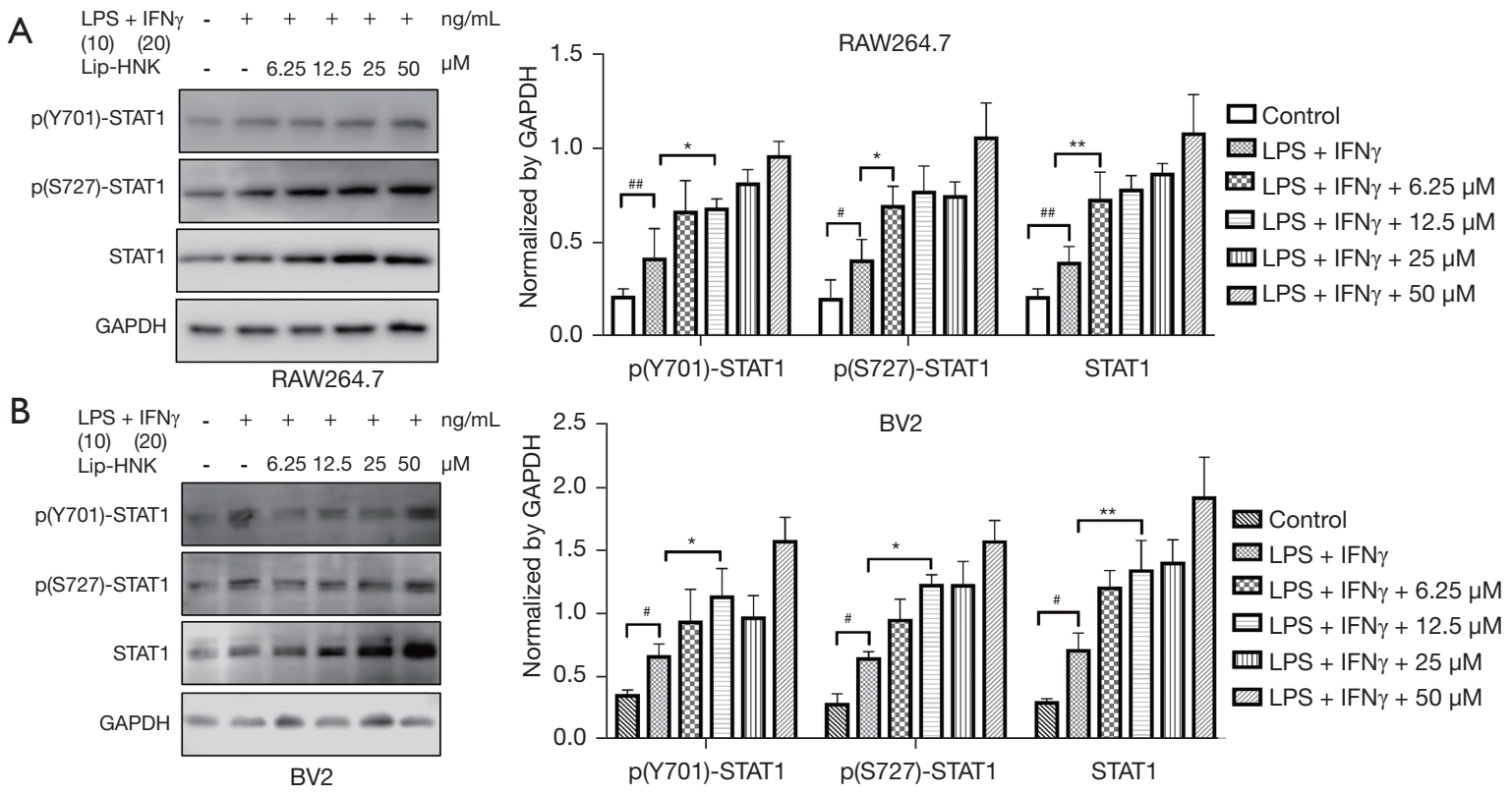

C
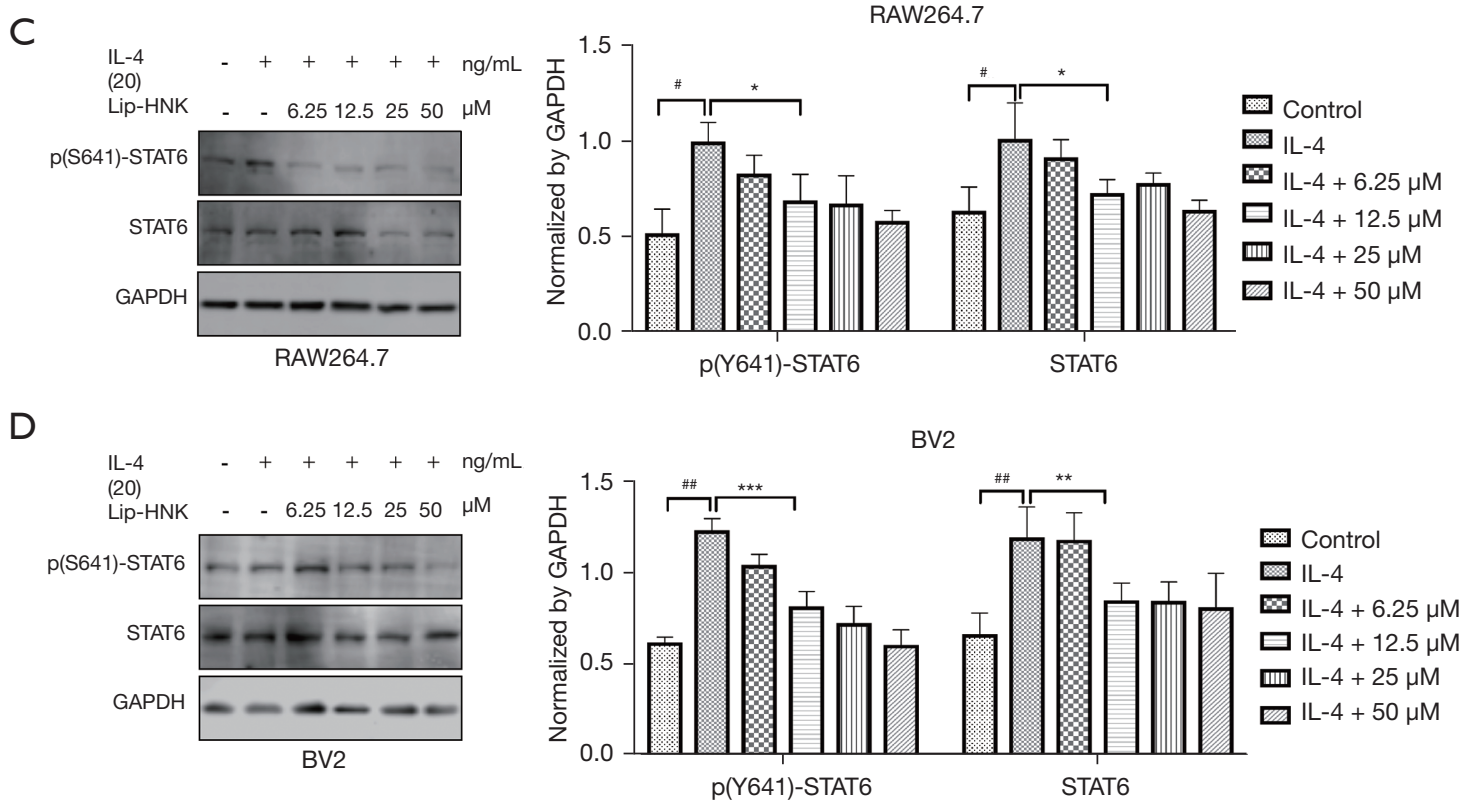

Figure 3 liposomal honokiol (Lip-HNK) regulates M1/M2 polarization via the STAT1/6 pathway. Interferon $\gamma$ (IFN- $\gamma$ ) $(20 \mathrm{ng} / \mathrm{mL})$ and lipopolysaccharide (LPS) $(10 \mathrm{ng} / \mathrm{mL})$ with Lip-HNK were used to treat RAW264.7 (A) and BV2 (B) cells for 48 hours. p-STAT1, activators of transcription (STAT1), and signal transducers p(Y701)-STAT1, p(S727)-STAT1, STAT1 were evaluated by Western blot. RAW264.7 (C) and BV2 (D) cells were treated with interleukin 4 (IL-4) $(20 \mathrm{ng} / \mathrm{mL})$ and indicated concentrations of Lip-HNK. p-STAT6 and STAT6 were evaluated using Western blot analysis. The 3 independent experiments are illustrated by histogram bars. The data are displayed as the mean \pm standard deviation. ${ }^{*}, \mathrm{P}<0.05 ;{ }^{* *}, \mathrm{P}<0.01 ;{ }^{* * *}, \mathrm{P}<0.001$. $^{*}, \mathrm{P}<0.05 ;{ }^{\# \#}, \mathrm{P}<0.01$. 

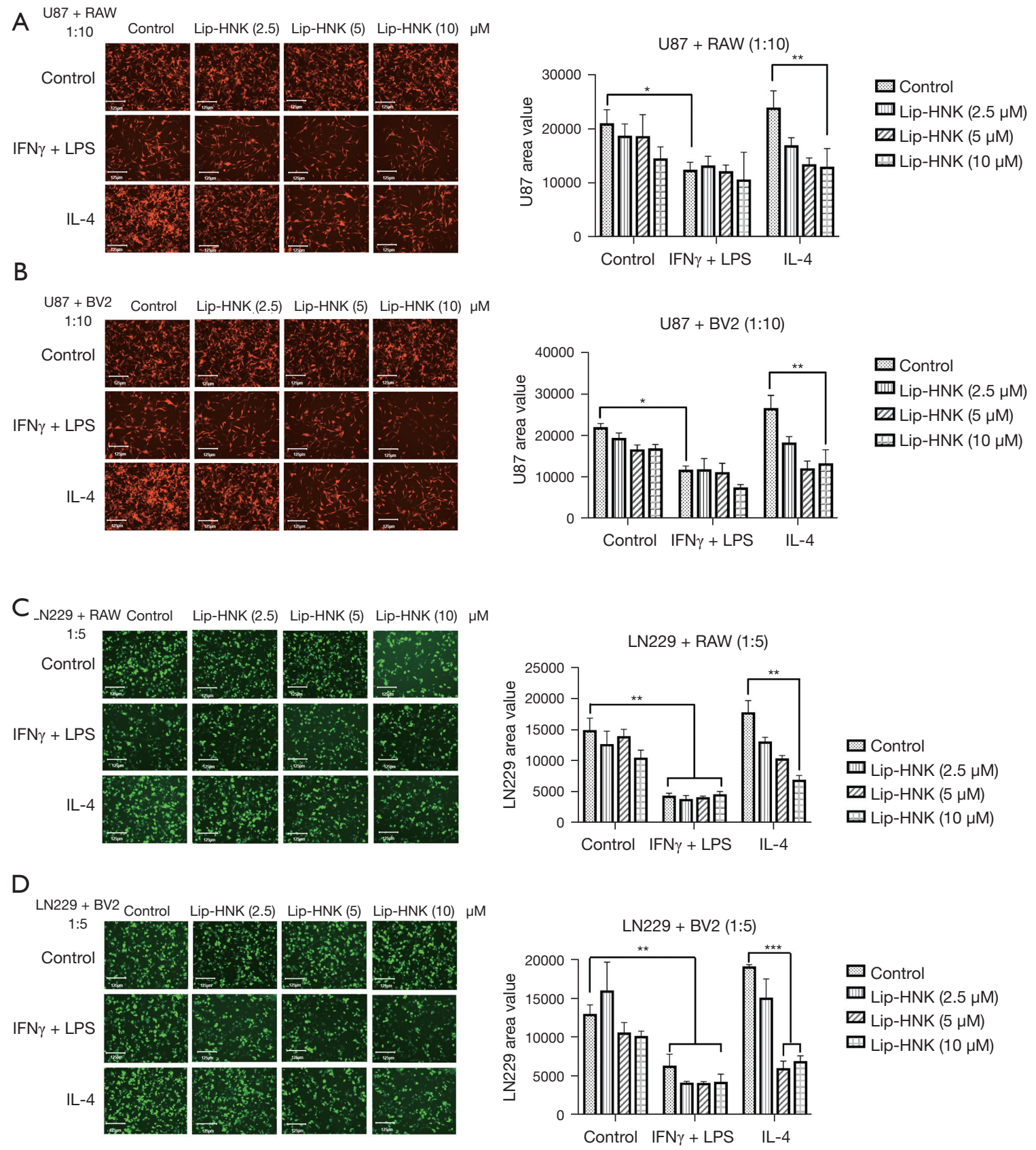

Figure 4 The impact of liposomal honokiol (Lip-HNK) on tumor cell proliferation in a co-culture environment with macrophages. Malignant tumor cells were co-cultured with either lipopolysaccharide (LPS) combined with interferon $\gamma$ (IFN- $\gamma$ ), interleukin 4 (IL-4) alone, or in a mixture with Lip-HNK at a fixed concentration for 48 hours. Co-culture of U87-RFP-Luc glioma cells was carried out with RAW264.7 (A) or BV2 (B) cells. The morphological features of the fluorescence-labeled tumor cells were examined under fluorescence microscopy, and the bioluminescence of glioma cells was detected after incubation with $15 \mu \mathrm{g} / \mathrm{mL}$ luciferase for $5 \mathrm{~min}$ using an EnSpire Multimode Plate Reader (PerkinElmer, Inc., USA). Fluorescent area or overall photon cell counts represented the proliferation of U87RFP-Luc cells using Image-Pro Plus software and the EnSpire Multimode Plate Reader. Co-culture of murine LN229-GFP glioma cells was carried out with RAW264.7 (C) or BV2 (D) cells. The growth of LN229-GFP cells was examined by the total fluorescent area of cells using Image-Pro Plus software. Three independent experiments are illustrated by the histogram bars. The data are displayed as the mean \pm standard deviation. * $\mathrm{P}<0.05 ;{ }^{* *}, \mathrm{P}<0.01 ;{ }^{* * *}, \mathrm{P}<0.001$. 

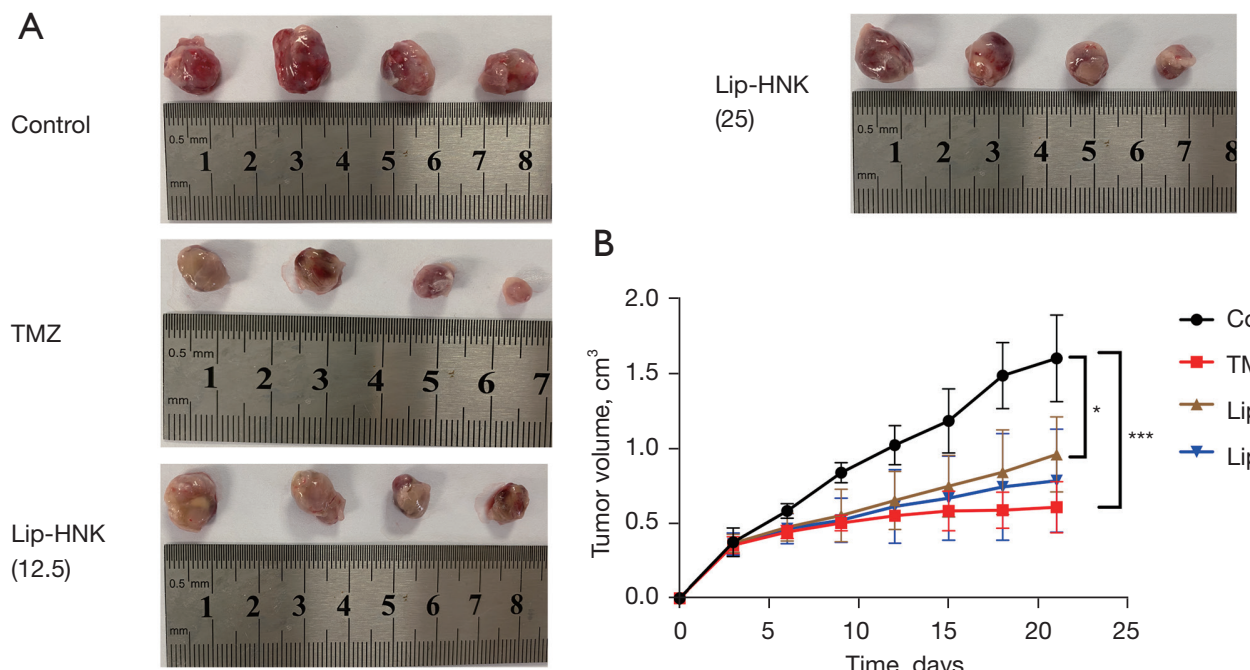

B

C

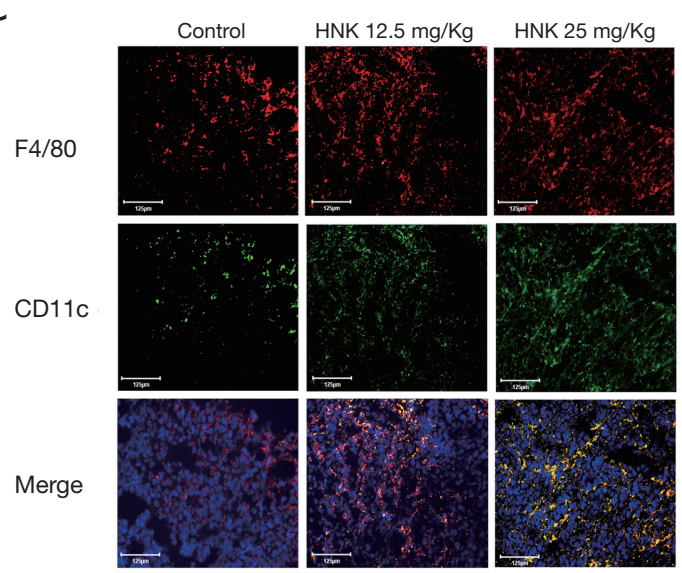

E
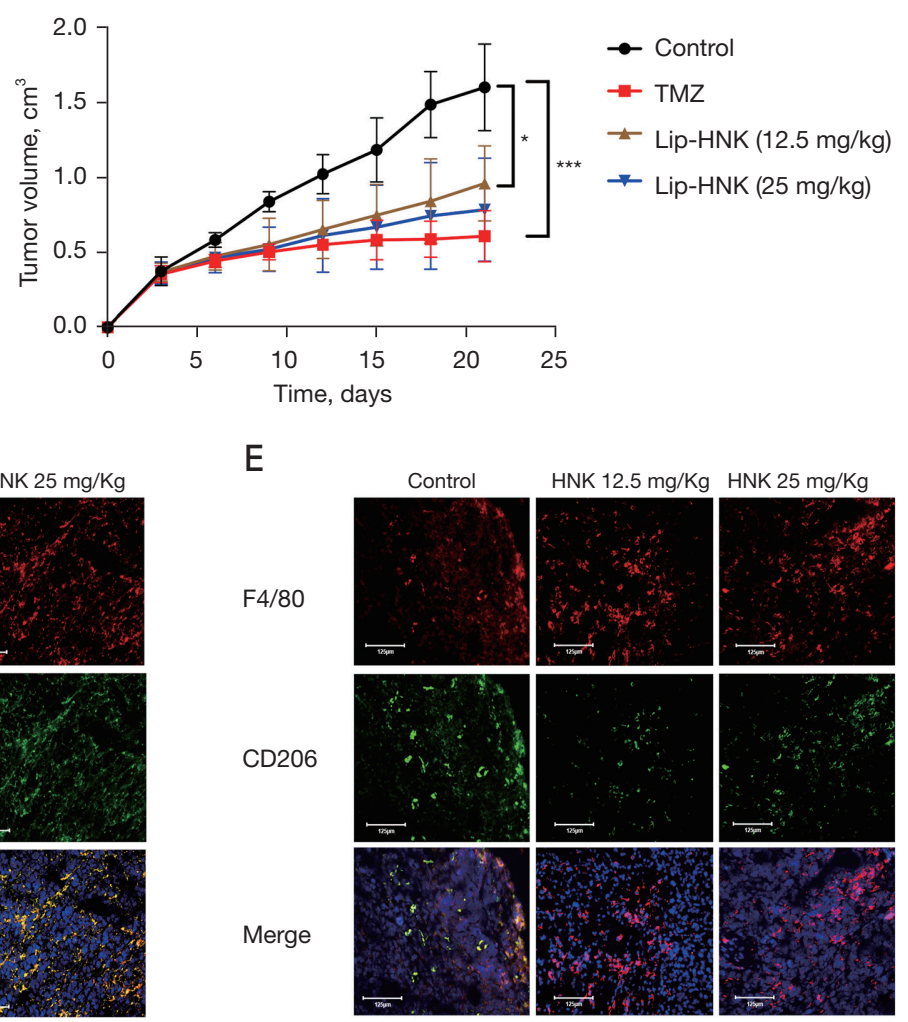

D

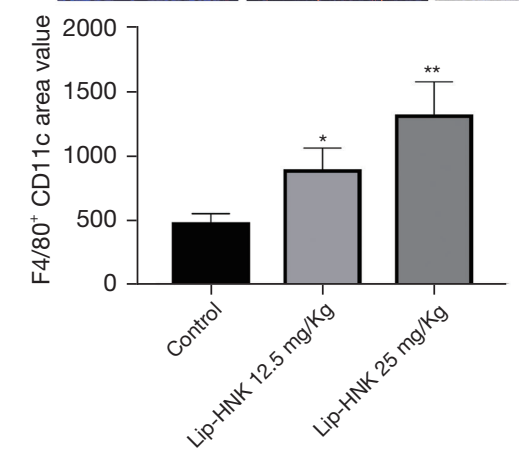

$\mathrm{F}$

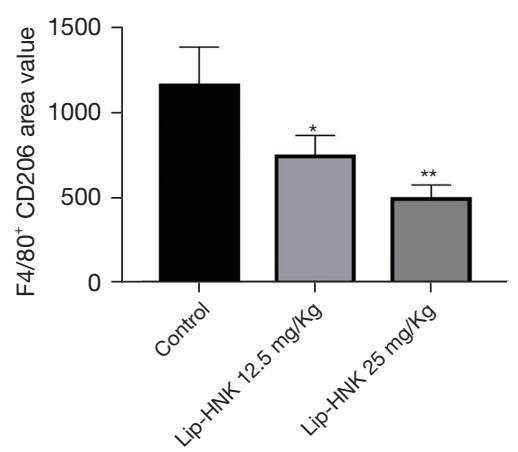

Figure 5 The effects of liposomal honokiol (Lip-HNK) on the progression of glioblastomas in a G422 xenograft model. G422 glioma cells were administered subcutaneously into the flanks of ICR mice, which were then treated with Lip-HNK (12.5 or $25 \mathrm{mg} / \mathrm{kg}$ per day) or temozolomide (TMZ) $(50 \mathrm{mg} / \mathrm{kg})$. The weights of the tumors (A) and tumor volume changes (B) were measured. Moreover, visualization of the infiltrating M1 macrophages in tumor sections was achieved using immunofluorescence staining of CD11c and F4/80 (C). Visualization of the tumor sections infiltrated by M2 macrophages was achieved using immunofluorescence staining of F4/80 and CD206 (D). The $\mathrm{F} 4 / 80^{+} \mathrm{CD} 11 \mathrm{c}^{+}$area $(\mathrm{E})$ and the $\mathrm{F} 4 / 80+\mathrm{CD} 206+$ area $(\mathrm{F})\left(\%\right.$ of $\mathrm{F} 4 / 80^{+}$tumor area) were quantified as the area of intersecting fields. Five randomly selected fields from mouse xenograft tumor sections were examined. *, $\mathrm{P}<0.05 ;{ }^{* *}, \mathrm{P}<0.01$; ***, $\mathrm{P}<0.001$. 
appropriate drug delivery system, such as liposomes, which are a new drug delivery system and provide a potential solution to this problem. Their unique phospholipid bilayer structure (similar to physiological membranes) makes them more compatible with the lipid bilayer of the BBB, which helps drugs enter the brain. Wang et al. (16) also showed that hyaluronic acid (HA)-modified cationic Lip-HNK is more effective than HNK. However, whether Lip-HNK can kill cancer cells and its potential anticancer mechanisms remain unclear.

Although Lip-HNK has entered phase I clinical trials for glioma treatment, its antitumor effect on glioma by regulating the phenotypic differentiation of macrophages has not been reported. This study is the first to demonstrate that Lip-HNK treatment inhibits the anti-inflammatory state of macrophages and induces activated macrophages to produce antitumor pro-inflammatory phenotypes, which is consistent with the suppression of GBM growth both in vivo and in vitro. At present, existing research on HNK mainly focuses on its toxicity on tumor cells. Other studies have also shown that HNK can suppress the growth of different types of malignant tumors, including GBM (17-19). One of the advantages of this study is the use of low concentrations of Lip-HNK. At the concentrations used here, we found no cytotoxicity in RAW264.7 and BV2 cells (Figure S1A). We provide new insights into the potential use of Lip-HNK as an antitumor drug, and we will further study the mechanisms and its specific effects on cancer. Our study found that Lip-HNK showed little direct toxicity on macrophages at concentrations below $10 \mu \mathrm{M}$. Therefore, in our study, Lip-HNK may inhibit the growth of gliomas by regulating the tumor microenvironment.

Studies have demonstrated that macrophages can repolarize completely from M2 to M1 and reverse their polarization according to the environment (20). In this study, for the polarization of M1, LPS and IFN- $\gamma$ were added to RAW264.7 and BV2 cells, which is the most recognized method of M1 polarization (21). Regarding the polarization of M2, IL-4, the most widely used M2 polarization method, was used for induction (22). Using these induction methods, we found that Lip-HNK can repolarize M2 macrophages to the M1 phenotype.

Polarization of M2 macrophages occurs in the microglia of malignant GBMs, which leads to immunosuppression. Therefore, it is reasonable to consider the inhibition of macrophage differentiation into the M2 phenotype as an important factor in escalating the immune response of patients suffering from GBM (23). The results of this study indicated that Lip-HNK treatment could inhibit M2 macrophage distribution ( $\mathrm{F} 4 / 80^{+} / \mathrm{CD} 206^{+}$cells) in the xenograft mouse model, and this phenomenon is in line with the decline of tumor masses seen in these models.

At the same time, we cannot deny this point, at least in vivo. Lip-HNK inducing the conversion of the M1 phenotype $\left(\mathrm{F} 4 / 80^{+}\right.$and $\left.\mathrm{CD} 11 \mathrm{c}^{+} / \mathrm{MHC}-\mathrm{II}-\mathrm{IA}-\mathrm{IE}^{+}\right)$may be a secondary effect in the treatment of GBM. Lip-HNK treatment reduced IL-4-induced expression of the antiinflammatory M2 markers CD206 and Arg1. Lip-HNK further increased the levels of iNOS, CD11c, and MHCII IA-IE induced by LPS/IFN- $\gamma$.

More and more evidence has shown that M1 polarized macrophages may be crucial antitumor agents, while M2 polarized macrophages are related to the growth, angiogenesis, migration, and invasion of malignant tumors (24-26). LPS/IFN- $\gamma$ treated macrophages could hinder both U87 and LN229 glioma cell growth. Lip-HNK could disrupt the growth of glioma cells as well as their interaction with M1 macrophages. On the contrary, the promotion of tumor cell growth was seen in macrophages treated with IL-4, and Lip-HNK has a satisfactory effect in inhibiting the proliferation of glioma and breast tumors induced by M2 macrophages. Overall, our data support that the antitumor effect of Lip-HNK in vivo and in vitro is dependent on macrophage polarization. We believe that Lip-HNK acts as an effective inhibitor of M2 activation and as a promoter of $\mathrm{M} 1$ activation. This present study provides new insights, as this is the first time that Lip-HNK has been shown to regulate immunity through regulating the polarization of M1/M2 macrophages. In particular, Lip-HNK inhibited M2 macrophage polarization and promoted M1 macrophage polarization. Previous literature has unveiled the specific signaling pathway responsible for the differentiation of macrophages. It has also been demonstrated that NF- $\kappa B$, the STAT family, the cAMP response element binding protein (CREB)-C/enhancer binding protein (EBP) axis, and nuclear receptor PPAR- $\gamma$ are involved in the polarization of macrophages $(27,28)$. In the preliminary data, Lip-HNK did not alter NF- $\mathrm{KB}$ activation (data not shown). The regulatory effect of NF$\kappa \mathrm{B}$ on macrophage polarization has remained controversial until the present study, which established that Lip-HNK could control macrophage M1/M2 polarization through the STAT1/6 pathways. In addition, the direct molecular mechanisms and targets of Lip-HNK in macrophages are worthy of further identification.

In addition, we also examined the impact of Lip-HNK 


\section{Page 12 of 13}

on several aspects of tumor biology, such as vessel density in mouse tissues. As shown in Figure S6, the expression of CD31 in the Lip-HNK group was significantly lower than that in the control group. It also suggests that Lip-HNK can inhibit tumor growth through various processes.

We also investigated the impact of hypoxic conditions on macrophages. RAW264.7 macrophage morphologies and phenotypes vary when cells are exposed to different hypoxic conditions. We cultured RAW264.7 cells for 7 days under normoxic or hypoxic conditions in the presence of LPS + IFN- $\gamma$ or IL-4. We found that hypoxia did not influence the expression of M1 macrophage-related markers in the LPS + IFN- $\gamma$ group, but it did increase the expression of M2 macrophage-related markers in the IL-4 group (Figure S7). The mechanism was not studied in our experiments. In the future, we will continue our investigations into these areas.

Finally, we showed that Lip-HNK can inhibit GBM growth both in vivo and in vitro, partly through the inhibition of M2 polarization and the promotion of M1 polarization, the mechanism of which may be through encouraging the activation of STAT1 and the inhibition of STAT6. In general, these findings suggest that LipHNK can achieve its anti-glioma function by mediating macrophage polarization. We provide new insights into the potential use of Lip-HNK as an antitumor drug, and we will further study the mechanisms and its specific effects on cancer. However, there were also some deficiencies in our study. We will apply orthotopic models for in vivo experiments for future studies, and experiments with $6-8$ or more mice per group will be employed. In addition, we will also see knockout STAT1 in the glioma animal model to confirm the elevated volume of glioma and the inactivation of M1 polarization compared with the wild-type glioma animal model.

\section{Acknowledgments}

We are thankful to our colleagues who provided their expertise that greatly assisted this research work.

Funding: This research was funded by The National Natural Science Foundation of China (grant number 81972338). This study was also financially funded by the Beijing Municipal Health Commission of China, Advanced Research and Training Program of Beijing Double Leading Scholars from China Academy of Chinese Medical Science, Clinical Major Specialty Projects of Beijing, and the National Science and Technology Major Project of China (No. 2016ZX09101017).

\section{Li et al. Modulation of antitumor immune responses by Lip-HNK}

\section{Footnote}

Reporting Checklist: The authors have completed the ARRIVE reporting checklist. Available at https://dx.doi. org/10.21037/atm-21-1836

Data Sharing Statement: Available at https://dx.doi. org/10.21037/atm-21-1836

Peer Review File: Available at https://dx.doi.org/10.21037/ atm-21-1836

Conflicts of Interest: All authors have completed the ICMJE uniform disclosure form (available at https://dx.doi. org/10.21037/atm-21-1836). The authors have no conflicts of interest to declare.

Ethical Statement: The authors are accountable for all aspects of the work in ensuring that questions related to the accuracy or integrity of any part of the work are appropriately investigated and resolved. All protocols and experiments in this study were approved by the Committee on the Ethics of Animal Experiments of Beijing Tiantan Hospital (approval number: KYSQ 2021-02101), in compliance with Chinese national and institutional guidelines for the care and use of animals.

Open Access Statement: This is an Open Access article distributed in accordance with the Creative Commons Attribution-NonCommercial-NoDerivs 4.0 International License (CC BY-NC-ND 4.0), which permits the noncommercial replication and distribution of the article with the strict proviso that no changes or edits are made and the original work is properly cited (including links to both the formal publication through the relevant DOI and the license). See: https://creativecommons.org/licenses/by-nc-nd/4.0/.

\section{References}

1. Lah TT, Novak M, Breznik B. Brain malignancies: Glioblastoma and brain metastases. Semin Cancer Biol 2020;60:262-73.

2. Chen Z, Hambardzumyan D. Immune Microenvironment in Glioblastoma Subtypes. Front Immunol 2018;9:1004.

3. Kvisten M, Mikkelsen VE, Stensjøen AL, et al. Microglia and macrophages in human glioblastomas: A morphological and immunohistochemical study. Mol Clin Oncol 2019;11:31-6. 
4. Dello Russo C, Lisi L, Tentori L, et al. Exploiting Microglial Functions for the Treatment of Glioblastoma. Curr Cancer Drug Targets 2017;17:267-81.

5. Sica A, Mantovani A. Macrophage plasticity and polarization: in vivo veritas. J Clin Invest 2012;122:787-95.

6. Shapouri-Moghaddam A, Mohammadian S, Vazini H, et al. Macrophage plasticity, polarization, and function in health and disease. J Cell Physiol 2018;233:6425-40.

7. Pyonteck SM, Akkari L, Schuhmacher AJ, et al. CSF$1 \mathrm{R}$ inhibition alters macrophage polarization and blocks glioma progression. Nat Med 2013;19:1264-72.

8. Katiyar SK. Emerging Phytochemicals for the Prevention and Treatment of Head and Neck Cancer. Molecules 2016;21:1610.

9. Pan J, Lee Y, Wang Y, et al. Honokiol targets mitochondria to halt cancer progression and metastasis. Mol Nutr Food Res 2016;60:1383-95.

10. Pan J, Lee Y, Zhang Q, et al. Honokiol Decreases Lung Cancer Metastasis through Inhibition of the STAT3 Signaling Pathway. Cancer Prev Res (Phila) 2017;10:133-41.

11. Tang WL, Tang WH, Szeitz A, et al. Systemic study of solvent-assisted active loading of gambogic acid into liposomes and its formulation optimization for improved delivery. Biomaterials 2018;166:13-26.

12. Robbins KS, Greenspan P, Pegg RB. Effect of pecan phenolics on the release of nitric oxide from murine RAW 264.7 macrophage cells. Food Chem 2016;212:681-7.

13. Lin JW, Chen JT, Hong CY, et al. Honokiol traverses the blood-brain barrier and induces apoptosis of neuroblastoma cells via an intrinsic bax-mitochondrioncytochrome c-caspase protease pathway. Neuro Oncol 2012;14:302-14.

14. Yeh PS, Wang W, Chang YA, et al. Honokiol induces autophagy of neuroblastoma cells through activating the PI3K/Akt/mTOR and endoplasmic reticular stress/ ERK1/2 signaling pathways and suppressing cell migration. Cancer Lett 2016;370:66-77.

15. Lin MC, Lee YW, Tseng YY, et al. Honokiol Induces Autophagic Apoptosis in Neuroblastoma Cells through a P53-Dependent Pathway. Am J Chin Med 2019;47:895-912.

16. Wang J, Liu D, Guan S, et al. Hyaluronic acid-modified liposomal honokiol nanocarrier: Enhance anti-metastasis and antitumor efficacy against breast cancer. Carbohydr Polym 2020;235:115981.

17. Shi X, Zhang T, Lou H, et al. Anticancer Effects of Honokiol via Mitochondrial Dysfunction Are Strongly Enhanced by the Mitochondria-Targeting Carrier Berberine. J Med Chem 2020;63:11786-800.
18. Wu GJ, Yang ST, Chen RM. Major Contribution of Caspase-9 to Honokiol-Induced Apoptotic Insults to Human Drug-Resistant Glioblastoma Cells. Molecules 2020;25:1450.

19. Yang J, Pei H, Luo H, et al. Correction: Non-toxic dose of liposomal honokiol suppresses metastasis of hepatocellular carcinoma through destabilizing EGFR and inhibiting the downstream pathways. Oncotarget 2020;11:3350-1.

20. Davis MJ, Tsang TM, Qiu Y, et al. Macrophage M1/M2 polarization dynamically adapts to changes in cytokine microenvironments in Cryptococcus neoformans infection. mBio 2013;4:e00264-13.

21. Das A, Sinha M, Datta S, et al. Monocyte and macrophage plasticity in tissue repair and regeneration. Am J Pathol 2015;185:2596-606.

22. Martinez FO, Helming L, Gordon S. Alternative activation of macrophages: an immunologic functional perspective. Annu Rev Immunol 2009;27:451-83.

23. Fujiwara Y, Komohara Y, Kudo R, et al. Oleanolic acid inhibits macrophage differentiation into the M2 phenotype and glioblastoma cell proliferation by suppressing the activation of STAT3. Oncol Rep 2011;26:1533-7.

24. Mantovani A, Biswas SK, Galdiero MR, et al. Macrophage plasticity and polarization in tissue repair and remodelling. J Pathol 2013;229:176-85.

25. Yan C, Yang Q, Gong Z. Tumor-Associated Neutrophils and Macrophages Promote Gender Disparity in Hepatocellular Carcinoma in Zebrafish. Cancer Res 2017;77:1395-407.

26. Locati M, Curtale G, Mantovani A. Diversity, Mechanisms, and Significance of Macrophage Plasticity. Annu Rev Pathol 2020;15:123-47.

27. Lawrence T, Natoli G. Transcriptional regulation of macrophage polarization: enabling diversity with identity. Nat Rev Immunol 2011;11:750-61.

28. Juhas U, Ryba-Stanisławowska M, Szargiej P, et al. Different pathways of macrophage activation and polarization. Postepy Hig Med Dosw (Online) 2015;69:496-502.

(English Language Editors: C. Betlazar-Maseh and J. Chapnick)

Cite this article as: $\mathrm{Li} \mathrm{S,} \mathrm{Li} \mathrm{L,} \mathrm{Chen} \mathrm{J,} \mathrm{Fan} \mathrm{Y,} \mathrm{Wang} \mathrm{C,}$ Du Y, Guo C, Chen F, Li W. Liposomal honokiol inhibits glioblastoma growth through regulating macrophage polarization. Ann Transl Med 2021;9(22):1644. doi: 10.21037/ atm-21-1836 


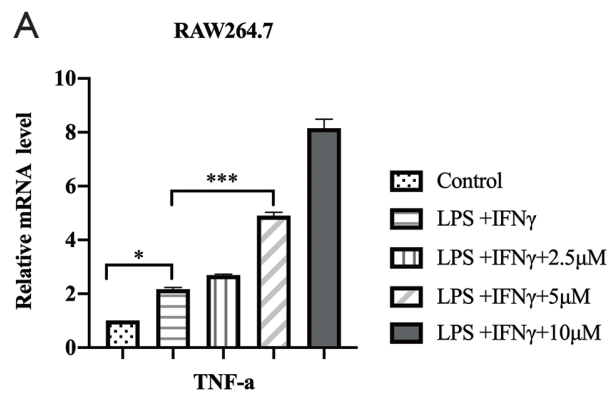

\section{B}

BV2

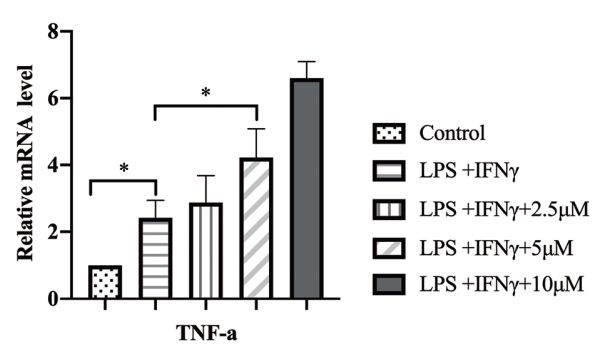

Figure S1 The effect of liposomal honokiol (Lip-HNK) on lipopolysaccharide (LPS) + interferon $\gamma$ (IFN- $\gamma$ )-mediated macrophage marker expression. The mRNA levels of tumor necrosis factor $\alpha(\mathrm{TNF}-\alpha)$ were detected by real-time reverse transcription polymerase chain reaction (RT-PCR) in RAW264.7 (A) and BV2 cells (B). *, P<0.05; ***, $\mathrm{P}<0.001$.
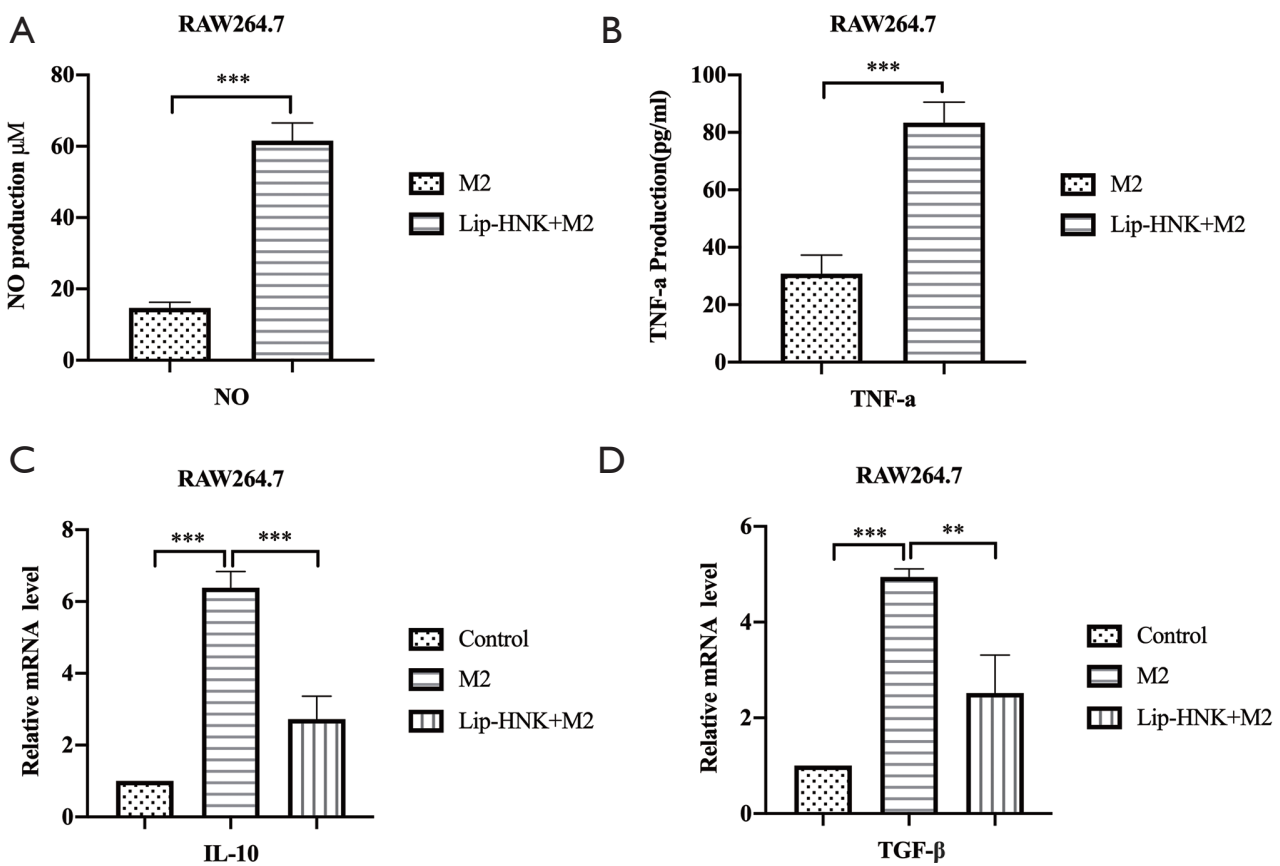

Figure S2 The levels of M1 and M2 related markers in RAW264.7 cells after induced with interleukin (IL)-4 and liposomal honokiol (LipHNK) treatment. The produced levels of nitric oxide (NO) (A), tumor necrosis factor $\alpha(\mathrm{TNF}-\alpha)(\mathrm{B})$, and the relative mRNA expression of IL-10 (C) and transforming growth factor $\beta($ TGF- $\beta$ ) (D) were detected by real-time reverse transcription polymerase chain reaction (RTPCR) in RAW264.7 cells. **, $\mathrm{P}<0.01$; ***, $\mathrm{P}<0.001$. 

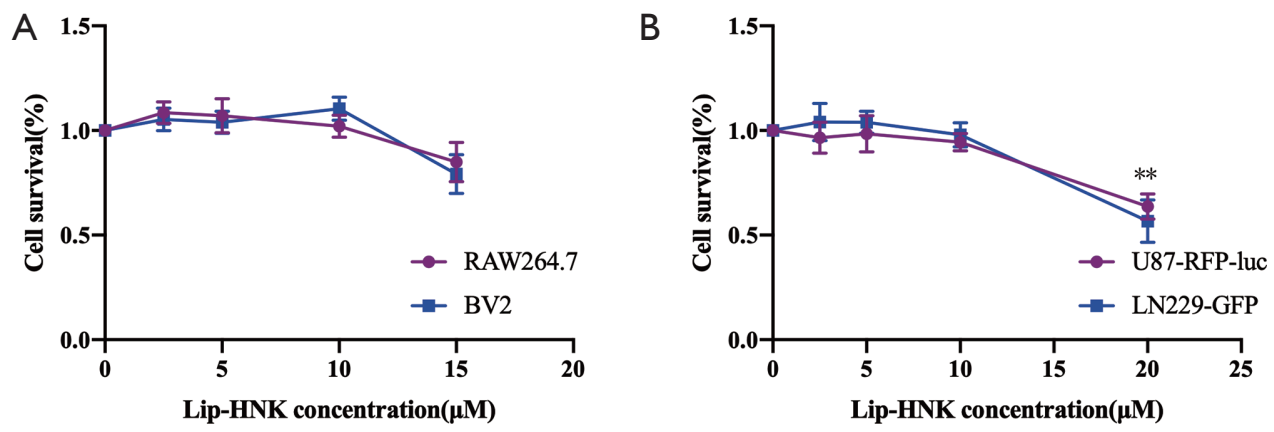

Figure S3 The effect of liposomal honokiol (Lip-HNK) on the proliferation of tumor, and macrophage cells. (A) Macrophage cells were treated with different concentration of Lip-HNK for 48 h; (B) U87 and LN229 tumor cell lines were treated with different concentration of Lip-HNK for 48 h. **, $\mathrm{P}<0.01$.

\section{U87-RFP-Luc cells}
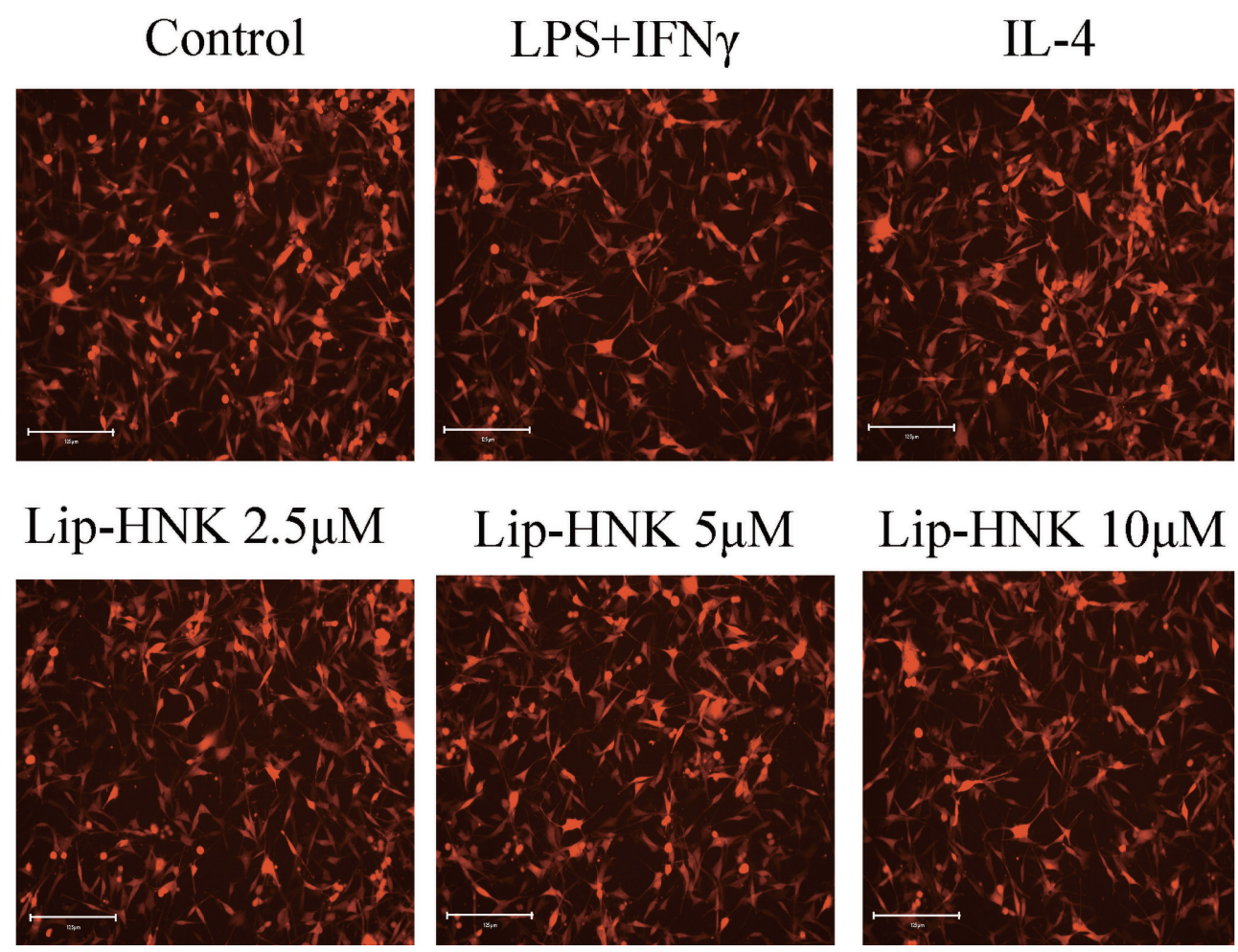

Figure S4 The effect of liposomal honokiol (Lip-HNK) and M1/M2 inducer on the proliferation of U87-RFP-Luc tumor cells. U87-RFPLuc glioma cells were treated with different concentration of Lip-HNK or interferon $\gamma$ (IFN- $\gamma$ )/lipopolysaccharide (LPS) and interleukin 4 (IL-4). The morphological feature of tumor cells was examined under fluorescence microscopy. 


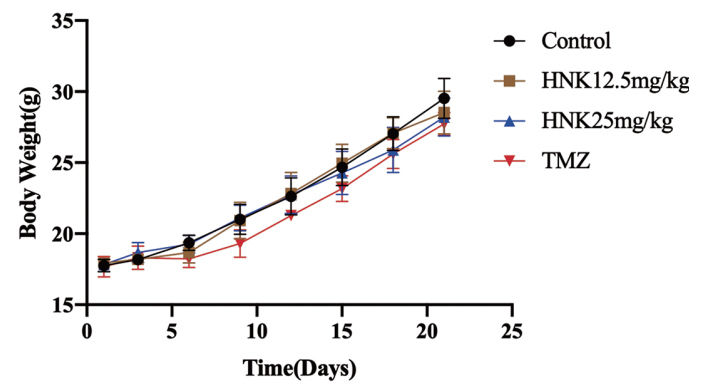

Figure S5 Effect of liposomal honokiol (Lip-HNK) on glioblastoma progression in G422 xenograft model. Body weight in individual mice were detected. TMZ, temozolomide.

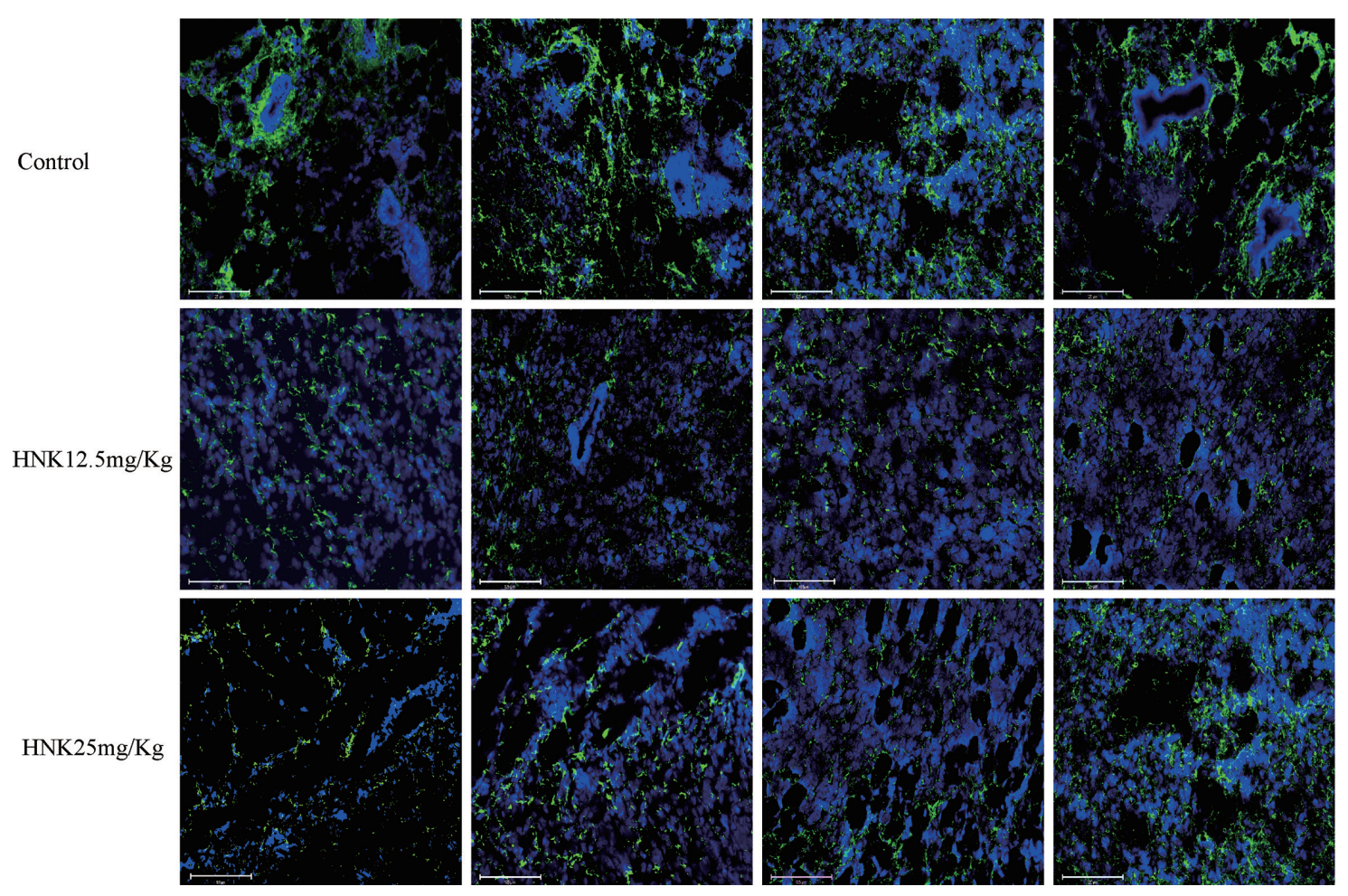

Figure S6 The expression of CD31 in liposomal honokiol (Lip-HNK) group and control group. Green fluorescence was used to detect the expression of CD31 in tumor tissue of G422 mice, blue fluorescence represents 4',6-diamidino-2-phenylindole (DAPI). 

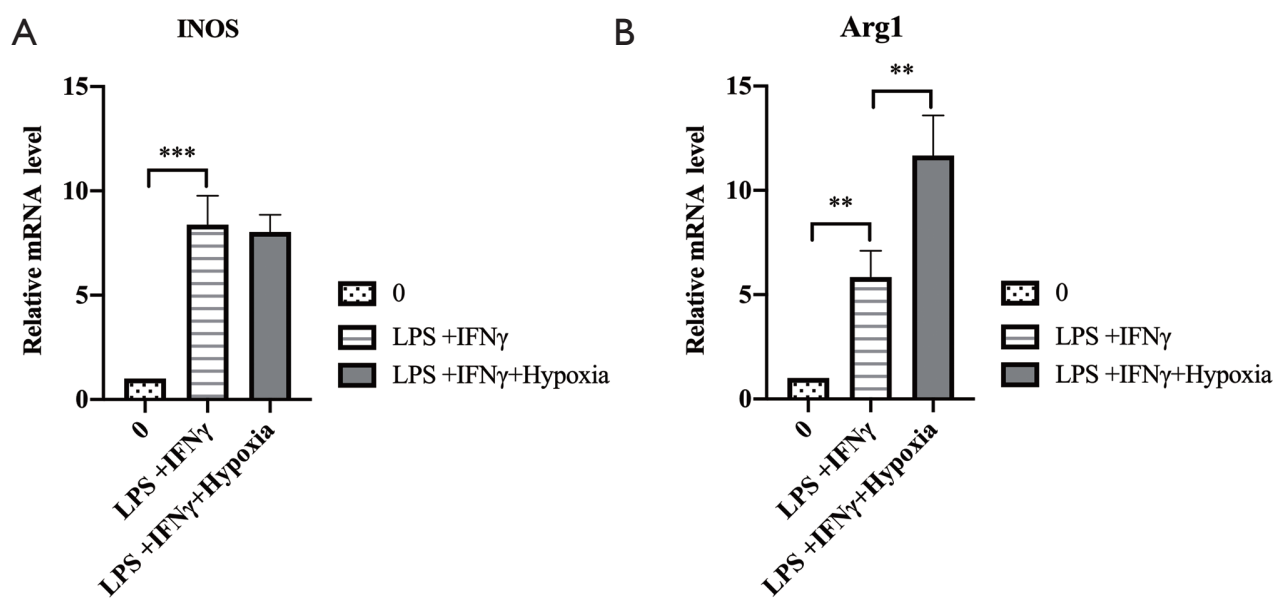

Figure S7 The expression of macrophage related markers in lipopolysaccharide (LPS) + interferon $\gamma$ (IFN- $\gamma$ ) (A) and interleukin 4 (IL-4) (B) group under hypoxia. **, $\mathrm{P}<0.01 ;{ }^{* * *}, \mathrm{P}<0.001$.

Table S1 The primer sequences used in the real time reverse transcription polymerase chain reaction (RT-PCR) analysis

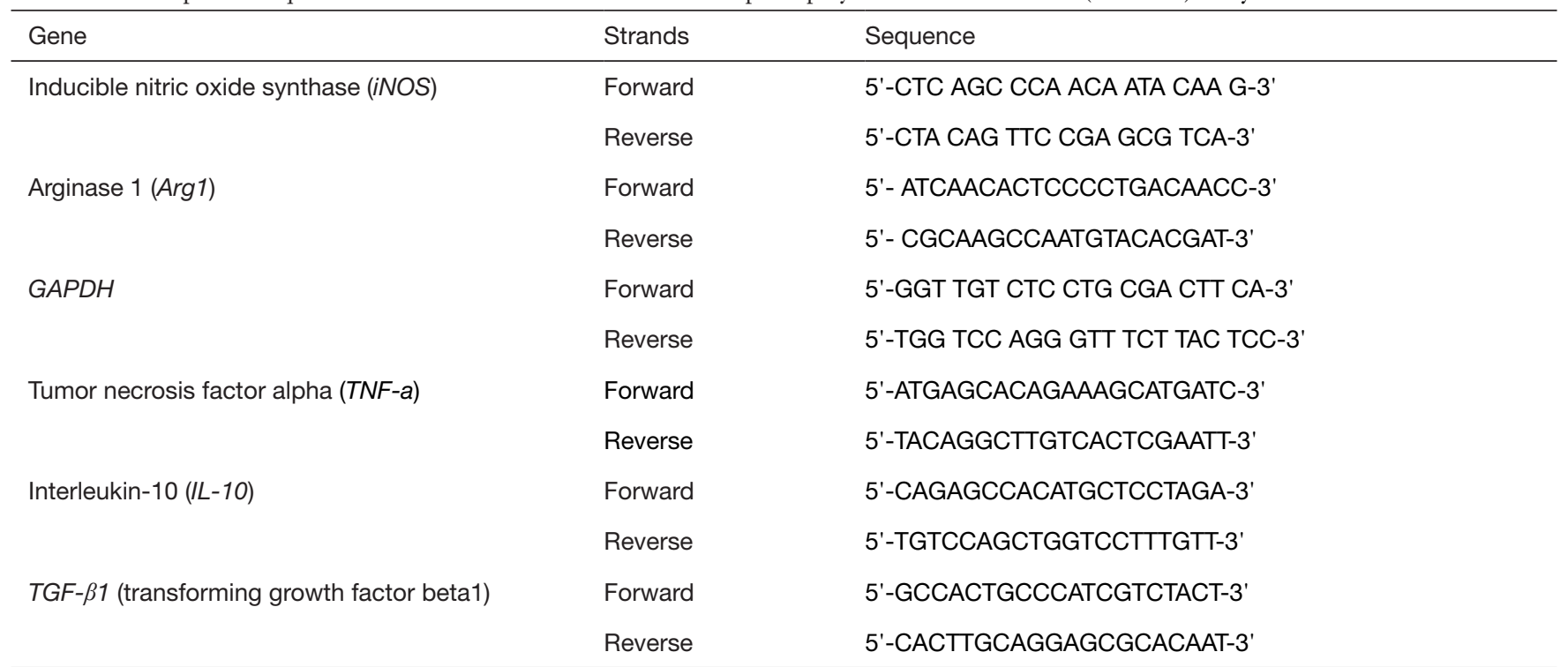

